\title{
Tomasz Bigaj®
}

\section{Counterparts, Essences and Quantified Modal Logic}

\begin{abstract}
It is commonplace to formalize propositions involving essential properties of objects in a language containing modal operators and quantifiers. Assuming David Lewis's counterpart theory as a semantic framework for quantified modal logic, I will show that certain statements discussed in the metaphysics of modality de re, such as the sufficiency condition for essential properties, cannot be faithfully formalized. A natural modification of Lewis's translation scheme seems to be an obvious solution but is not acceptable for various reasons. Consequently, the only safe way to express some intuitions regarding essential properties is to use directly the language of counterpart theory without modal operators.
\end{abstract}

Keywords: quantified modal logic; counterpart theory; essentialism; cheap haecceitism; David Lewis

\section{Introduction}

The problem considered below is mainly logical in character, even though it is motivated by some metaphysical debates. It can be concisely expressed as the question of how to formalize certain intuitive statements regarding representation de re and essential properties of objects in the language of quantified modal logic. David Lewis in his 1968 article (reprinted in [Lewis 1983] with a postscript) has proposed a possibleworld semantics for quantified modal logic which is based on the notion of a counterpart relation. I will assume his counterpart theory, and I will show that some oft-used metaphysical statements expressed in terms of possible worlds and counterparts cannot be properly translated into familiar quantified modal sentences. I will discuss a natural modification of Lewis's counterpart theory which can solve the translatability problem, and I will show that it creates more problems in other areas. 
The conclusion of this analysis will be largely negative: it seems that quantified modal logic is incapable of expressing some rather important statements regarding properties of the counterparts of actual objects in possible worlds. ${ }^{1}$

\section{Lewis's translation scheme}

Lewis's counterpart theory is expressed in a first-order language whose primitive predicates are as follows:

- one-place predicate $W$ ("is a world"),

- one-place predicate $A$ ("is actual"),

- two-place predicate $I$ ("is in a world"),

- two-place predicate $C$ ("is a counterpart of").

The predicates introduced above are assumed to satisfy a set of postulates, which we don't have to write down here [but see Lewis 1983, p. 27]. However, it is important to remember that the counterpart relation expressed by $C$ is not assumed to be one-to-one even if its domain is restricted to one world; i.e., an object can possess more than one counterpart in a given world, and two objects from one world can have the same counterpart in another. Moreover, it is stipulated that every actual object is its own counterpart, and that there are no other counterparts of a given object in the actual world (this restriction is lifted in the later variant of Lewis's theory known as "cheap haecceitism" - see Sec. 5 for details).

Lewis then formulates a recursive definition of translations from the set of well-formed formulas of quantified modal logic, i.e., first order logic without constants but with the modal operators $\square$ and $\diamond$, into the language of his counterpart theory. Again, we don't need to invoke the entire translation scheme here, except for the special case of open formulas with one free variable prefixed with either $\square$ or $\diamond$. Let $\varphi(\alpha)$ be a formula whose only free variable is $\alpha$. In that case the Lewisian translations are the following:

(2.1) for $\square \varphi(\alpha)$ the translation is: $\forall \beta \forall \gamma\left[(W \beta \wedge I \gamma \beta \wedge C \gamma \alpha) \rightarrow \varphi^{\beta}(\gamma)\right]$,

1 For a brief survey of some other problems related to quantified modal logic [see Garson 2018, sec. 15] and the bibliography therein. Of course, in this brief note I won't be able to do justice to all the work on quantified modal logic that has been done since Lewis's 1968 paper. 
(2.2) for $\diamond \varphi(\alpha)$ the translation is: $\exists \beta \exists \gamma\left[\left(W \beta \wedge I \gamma \beta \wedge C \gamma \alpha \wedge \varphi^{\beta}(\gamma)\right)\right]$, where $\varphi^{\beta}(\gamma)$ is a formula which results from $\varphi(\alpha)$ by replacing all occurrences of $\alpha$ by $\gamma$, and by restricting all its quantifiers to the domain of objects in world $\beta$ (i.e., we replace $\forall \delta \ldots$ with $\forall \delta(I \delta \beta \rightarrow \ldots)$, and $\exists \delta \ldots$ with $\exists \delta(I \delta \beta \wedge \ldots))$. Informally, we can say that according to the rule of translation presented in (2.1), formula $\square \varphi(\alpha)$ is true of any object $a$ in the actual world, if formula $\varphi(\alpha)$ is true of any counterpart of $a$ in any possible world. Analogously for (2.2): $\nabla \varphi(\alpha)$ is true of $a$ if $\varphi(\alpha)$ is true of some counterparts of $a$ in some possible worlds.

Lewis observes that his framework enables us to introduce the concept of an essential property for an object. This can be done as follows: a one-place open formula $\varphi(x)$ represents an essential property of an object $a$, iff $\square \varphi(x)$ is true of $a$. Alternatively, we may express this condition in the following, logically equivalent way. The formula $\varphi(x)$ denotes an essential property of $a$ iff the formula

$$
\square \forall y[y=x \rightarrow \varphi(y)]
$$

is true of $a$ (necessarily, whatever is identical with $a$, possesses the property denoted by $\varphi) .^{2}$ In the framework of counterpart theory this means that if $\varphi$ is an essential property of $a, \varphi$ is possessed by all the counterparts of $a$ in all possible worlds. This characterization meshes well with the standard description of essential properties as properties whose possession is necessary for being a given object. ${ }^{3}$ However, some insist that the complete essential properties of object $a$ should form not only

2 Penelope Mackie questions the correctness of expression $\square \varphi(x)$ as a formalization of essential properties [Mackie 2006, p. 5]. She insists that this formula carries the implication of the necessary existence of an object satisfying $\varphi$ (i.e., the existence of its counterparts in all possible worlds), which is not part of what we mean by possessing essential properties. In order to deal with this problem, she suggests using the conditional formula $\square(x$ exists $\rightarrow \varphi(x))$ instead. While she does not explain the presupposed interpretation of the predicate "exists" in this context, one way to explicate it could be with the help of the existential quantifier as follows: $\square(\exists y y=x \rightarrow \varphi(x))$. However, the formula under the operator of necessity is just logically equivalent to $\varphi(x)$. Note that in Lewis's approach the problem that worries Mackie does not arise, since the translation of formula $\square \varphi(x)$ is already conditional: $\forall \beta \forall y[(W \beta \wedge I y \beta \wedge C y x) \rightarrow \varphi(y)]$, and thus may remain true of a given object $a$ even if $a$ does not have a counterpart in some worlds.

3 We should mention here a well-known critique of the modal account of essential properties by Kit Fine [1994]. Fine famously observes that definition (2.3) implies for instance that the property of being a member of the singleton \{Socrates\} is an essential property of Socrates, since it is necessary that Socrates $\in$ \{Socrates $\}$. I don't have 
a necessary but also sufficient condition for being $a$. This is associated with the idea of an individual essence - a property (or a set of properties) that identifies uniquely an object in question under all possible circumstances. ${ }^{4}$

A natural way of characterizing an essential property that forms an individual essence is with the help of the sufficiency condition (in addition to the necessity condition (2.3) stated above), which is typically presented as

$$
\square \forall y[\varphi(y) \rightarrow y=x] .^{5}
$$

It seems that the satisfaction of this formula by an actual object $a$ implies that, first, $a$ is the only actual object (if any) possessing property $\varphi$ and, second, in all possible worlds whatever possesses $\varphi$ is guaranteed to be (a counterpart of) $a$. But Lewis's translation rule (2.1) does not support this reading. The translation produces a rather complex formula with one free variable $x$ which states that for any counterpart $\gamma$ of $\alpha$ in any possible world $\beta$, if anything in $\beta$ possesses $\varphi$, it is identical in $\beta$ to $\gamma$. Formally:

$$
\forall \beta \forall \gamma((W \beta \wedge I \gamma \beta \wedge C \gamma x) \rightarrow \forall y[\operatorname{Iy} \beta \rightarrow(\varphi(y) \rightarrow y=\gamma)])
$$

space to discuss this problem in detail here but I don't think that it damages the modal conception of essential properties beyond repair. The key point to be made here is that formula (2.3) defines an essential property under the condition that formula $\varphi(x)$ itself represents a genuine property. Virtually no modern metaphysician accepts the unrestricted version of realism, according to which all meaningful predicates correspond to genuine, real properties. The "property" of being a member of the singleton \{Socrates\} belongs to the category of so-called "impure" properties, since it involves an individual: Socrates. I side with those metaphysicians who believe that impure properties are not genuine properties at all, and therefore the problem whether being an element of \{Socrates\} is an essential property of Socrates does not even arise. Thus our analysis of essential properties should be explicitly conditional: if $\varphi(x)$ represents a genuine (i.e., pure) property $P$, then $P$ is essential iff (2.3) obtains.

4 According to Mackie, "[a]n individual essence of an object A would be a property, or set of properties, that is necessarily both necessary and sufficient for being A" [Mackie 2006, p. 19].

${ }^{5}$ Cf. (Glick 2016) where he defines his Sufficiency Condition using the exact same formula. It has to be noted, though, that he is aware of a problem with this definition when more than one counterpart in a world is admitted. More specifically, he claims that the truth of Sufficiency Condition prevents the existence of distinct counterparts in a given world. Later in the main text we will consider an alternative interpretation which enables Sufficiency to be true without excluding multiple counterparts. 
Suppose that the above formula is satisfied by an actual object $a$. The stated condition means that if in a given possible world $\beta$ there are counterparts of $a$, and there is an object $b$ satisfying $\varphi$, the following facts obtain: (i) $b$ is a counterpart of $a$, (ii) there is no object in $\beta$ that satisfies $\varphi$ other than $b$, and (iii) there is no counterpart of $a$ in $\beta$ other than $b$. In other words: for every world $\beta$, either there is no object in $\beta$ satisfying $\varphi$, or there is no counterpart of $a$ in $\beta$, or else there is exactly one counterpart of $a$ in $\beta$ that also satisfies $\varphi$. But this is not what we intuitively mean by the sufficiency condition. The intuition behind this concept is that whatever possible object possesses the property denoted by $\varphi$, this object is guaranteed to be a counterpart of our initially considered actual object. But condition (2.5) remains satisfied by an actual object $a$ if there is a possible world full of $\varphi$ possessing non-counterparts of $a$, if only $a$ has no counterpart there whatsoever. Thus the 'sufficiency' of possessing property $\varphi$ for being a given object, as expressed in (2.5), is rather peculiar. ${ }^{6}$

\section{Modified translation scheme}

The 'proper' sufficiency condition expressed in the language of counterpart theory is straightforward:

$$
\forall \beta \forall \gamma[(W \beta \wedge I \gamma \beta \wedge \varphi(\gamma)) \rightarrow C \gamma \alpha]
$$

However, its reverse translation into the language of quantified modal logic is far from obvious - as we have seen above, the formula $\square \forall y[\varphi(y)$ $\rightarrow y=x]$, though natural, won't do. As Lewis points out, not every statement in counterpart theory has its equivalent in quantified modal

${ }^{6}$ One may point out that there is yet another intuitive way of reading formula $\square \forall y[\varphi(y) \rightarrow y=x]$ which diverges slightly from the "sufficiency condition" as presented above. The formula under the necessity operator expresses the fact that there is at most one object in the domain which satisfies $\varphi$ (or, more accurately, the formula is satisfied by an object $a$, iff there is at most one object satisfying $\varphi$, and this object is $a$ ). Thus, it may be suggested that the 'necessitated' variant of this non-modal situation simply extends it to all possible worlds as follows: the modal formula $\square \forall y[\varphi(y) \rightarrow$ $y=x]$ is satisfied by $a$ iff in every possible world there is at most one object satisfying $\varphi$, and this object (if it exists) is a counterpart of $a$. But, as we have seen above, this interpretation does not accord with Lewis's translation (2.5) either. Lewis's translation admits the possibility that in a given possible world there may be more than one object satisfying $\varphi$, if only this world does not contain any counterpart of $a$. 
logic. While I don't have a formal proof for this contention, it seems rather clear to me that there can't be a modal quantified formula whose translation in counterpart theory is precisely the above statement (but see a simple formal argument for this claim under the additional assumption of cheap haecceitism given in Sec. 5). However, the situation may change if we modify slightly the Lewisian rules of translation, as given in (2.1) and (2.2). The suggested modification will be such that, before applying any of these rules, we should replace formula $\varphi(\alpha)$ with its modified variant $\varphi^{*}(\alpha)$ defined as follows:

(3.2) $\varphi^{*}(\alpha)$ is obtained from $\varphi(\alpha)$ by replacing all identities of the form $\alpha=\gamma$, where $\gamma$ is bound by a quantifier, with $C \gamma \alpha{ }^{7}$

The motivation behind this replacement should be obvious. Identities of the form $\alpha=\gamma$ connect variable $\alpha$, which is free and therefore supposed to range over actual objects, with a bound variable $\gamma$, which when quantified over under the modal operators $\square$ or $\diamond$ is assumed to range over objects in some possible world. But we know that in Lewis's approach objects inhabiting distinct worlds cannot be connected by numerical identity - the only transworld relation close to identity is the counterpart relation - hence the replacement ${ }^{8}$. On the other hand, identities that connect variables restricted to one and the same world (whether actual or merely possible) can be seen as denoting genuine numerical identity, and thus may be left untouched.

Once we have substituted formula $\varphi^{*}(\alpha)$ for $\varphi(\alpha)$, we have to apply the modified variants of translations (2.1) or (2.2). The modification is the following: formula $\varphi^{\beta}(\gamma)$ in translations given in $(2.1)$ and $(2.2)$ is replaced by $\varphi^{* \beta}(\gamma)$, where the latter is the result of first substituting all the occurrences of $\alpha$ by $\gamma$ in $\varphi^{*}(\alpha)$ except the occurrences in expressions

7 This is modeled on the proposal presented in [Bigaj 2016, p. 157]. To avoid possible misunderstandings, we should stress that (3.2) is the first step of a new translation procedure that leads from the language of quantified modal logic to the language of the counterpart theory, and not a pre-translation replacement of the original formula with a new one that subsequently will be translated using Lewis's rules. So a possible objection that the proposed translation scheme is not reductive, since it already presupposes the use of the counterpart-theoretical term $C$, is misguided.

8 Of course the counterpart relation is much more metaphysically loaded than numerical identity, which is in a sense trivial. 
of the form $C \gamma \alpha .^{9}$ Then the usual restriction of all the quantifiers in $\varphi^{*}(\gamma)$ to the domain of world $\beta$ follows.

Let us apply this new translation method to formula (2.4). Its starred counterpart according to $(3.2)$ will be $\forall y[\varphi(y) \rightarrow C y x]$. Thus the modified counterpart-theoretical translation of the entire modal formula (2.4) is as follows:

$$
\forall \beta((W \beta \rightarrow \forall y[(\operatorname{Iy} \beta \wedge \varphi(y)) \rightarrow C y x])
$$

which is exactly the counterpart-theoretical sufficiency condition as stated in (3.1). The modified translation scheme also produces the right result with respect to the 'necessity' condition $\square \forall y[y=x \rightarrow \varphi(y)]$, since its translation will be:

$$
\forall \beta((W \beta \rightarrow \forall y[(\operatorname{Iy} \beta \wedge C y x) \rightarrow \varphi(y)])
$$

which means that all counterparts of $x$ satisfy formula $\varphi$ in all possible worlds, as expected.

Unfortunately, in spite of its initial successes the modified translation scheme which treats non-uniformly identity formulas depending on the type of variables connected and their position with respect to modal operators, produces highly unintuitive interpretations of some modal expressions. Worse still, it may be claimed that the resulting modal logic is inconsistent, as it appears to violate some basic rules of inference. Let us start with a mild case of intuition violation first. Suppose that the formula to be translated is the following:

$$
\square \forall y[\varphi(x, y) \rightarrow y=x]
$$

Intuitively, this formula states that necessarily, $x$ stands in relation denoted by $\varphi$ to itself only, if it stands to anything at all. Thus we would expect this formula to be satisfied by some object $a$ in the case when in all possible worlds, for all counterparts of $a$ either they don't stand in $\varphi$ to anything in their respective worlds, or they stand in $\varphi$ to themselves and nothing else (in short: no counterpart of $a$ is related by $\varphi$ to any object other than itself). However, this intuition is not preserved under the currently adopted rules of translation. The modified counterparttheoretical translation of the above formula (3.5) is as follows:

${ }^{9}$ If formula $\varphi^{*}(\alpha)$ does not contain any occurrence of variable $\alpha$ except in expressions $C \gamma \alpha$, then the entire translation has the simplified form $\forall \beta\left(W \beta \rightarrow \varphi^{* \beta}(\alpha)\right)$ or $\exists \beta\left(W \beta \wedge \varphi^{* \beta}(\alpha)\right)$. 


$$
\forall \beta \in W \forall \gamma((I \gamma \beta \wedge C \gamma x) \rightarrow \forall y[\operatorname{Iy} \beta \rightarrow(\varphi(\gamma, y) \rightarrow C y x)])
$$

The meaning of the above formula is that it is satisfied by $a$ in the case when, for every possible world and every counterpart of $a$ in $\beta$, if this counterpart stands in relation $\varphi$ to any object, this object is also a counterpart of $a$. In other words, the relation denoted by $\varphi$ connects counterparts with counterparts only. This interpretation agrees with the intuitive one only when objects have at most one counterpart in any possible world. But in the case of multiple counterparts, the two interpretations diverge. ${ }^{10}$

More troublesome are examples in which well-known rules of inference for modal logic appear to be violated. Let us consider first the tautological formula $\exists y(y=x)$. Its modal version $\square \exists y(y=x)$ seems to express the thought that in all possible worlds and for any arbitrary object $x$ there is an object identical with it, which seems trivially true. But observe that under the current proposal we should interpret the identity $y=x$ under the existential quantifier as connecting objects from distinct worlds, since one variable is free while the other bound. Hence the meaning of the considered formula is that an arbitrary actual object $x$ possesses its counterpart in any possible world, which does not seem to be true, let alone logically true.

This consequence by itself may not be seen as catastrophic (and some would even claim that it is beneficial), since the intuitions regarding modal statements are shaky, and as we have already observed it is at least feasible to interpret the modal formula $\square \exists y(y=x)$ as stating just that that objects have counterparts in every possible world (see Lewis's own remarks on that problem in [Lewis 1983, p. 31]). However, there is more trouble ahead. When we consider the closed formula $\square \forall x \exists y(y=x)$, we note that the interpretation of the identity operator must change, since now it connects two bound variables, and hence variables whose ranges are limited to one and the same possible world. Consequently, the entire sentence becomes logically true (it is logically guaranteed that in every possible world and for every object there is something identical with it namely the object itself). But this means that we have to abandon the following rule of inference:

10 Interestingly, in this case Lewis's original translation schema produces the required result, in contrast to the previously considered case of "Sufficiency Condition" (ft. 5). 


$$
\frac{\square \forall x \varphi(x)}{\square \varphi(x)}
$$

which enables us to derive from the necessity of a closed universally quantified sentence the necessity of the open formula under the quantifier.

Here is another bit of bad news for the modified translation scheme currently under discussion. As is well known, the open formula $\varphi(x)$ is logically equivalent to $\exists y[y=x \wedge \varphi(y)]$. But now consider translations of these two formulas prefixed by the operator of necessity $\square$. The first one is straightforward - as we have already observed it means that for every counterpart of $x$ in every possible world, this counterpart satisfies $\varphi$. But with the second formula the translation is markedly different: it states that in every possible world there is a counterpart of $x$ which possesses property $\varphi$. The problem with this translation is not only that it implies that in every possible world there is a counterpart of $x$ (and hence that every object satisfying the original formula must exist necessarily - this is what worried Mackie, as explained in ft. 2 above), but first and foremost that it allows for the existence of counterparts that don't satisfy $\varphi$, which is clearly precluded by formula $\square \varphi(x)$. The formulas $\square \varphi(x)$ and $\square \exists y[y=x \wedge \varphi(y)]$ become equivalent only under the assumption that in every possible world there is exactly one counterpart of any actual object (this assumption is close in spirit to the controversial claim promulgated by Ruth Barcan Marcus that all possible worlds have the exact same domain). But if we, following Lewis, admit possible worlds with no counterparts of a given actual object, and possible worlds with more than one counterpart of the same object, then both formulas diverge. Consequently, there is a breach of the rule which enables us to replace salva veritate any subformula of a given formula by its logical equivalent. The formulas $\varphi(x)$ is and $\exists y[y=x \wedge \varphi(y)]$ are logically equivalent, and yet $\square \varphi(x)$ and $\square \exists y[y=x \wedge \varphi(y)]$ can differ with respect to their truth values (i.e. satisfaction).

\section{Possible ways out}

The remarks made in the last paragraph hint at one possible solution of the problem affecting the modified translation scheme. As we have already observed, all the controversial examples discussed earlier dissolve when we assume that every actual object has precisely one counterpart in any possible world. This follows from the simple fact that under 
this assumption the modified translation scheme is equivalent to the original, Lewisian one. The conditionals of the form $C \gamma x \rightarrow \ldots \gamma=z \ldots$ in Lewis's translation formula (2.1) are replaced by the unconditional $\ldots C x z \ldots$ in the modified method but they become equivalent provided that for any object $x$ and in any possible world there is exactly one $\gamma$ such that $C \gamma x$. Unfortunately, this solution is far from being uncontroversial. It goes against our basic modal intuitions to claim that every actual object exists necessarily, i.e. in any possible world. I can clearly imagine a possible world in which luckily there was neither Hitler nor Stalin. To insist that their existence must be necessary offends not only our modal, but perhaps also moral intuitions.

A slightly better line of defense is based on the elementary observation that identity statements in modal contexts are notoriously ambiguous. We have already noted that intuitions associated with modal formulas involving identities may vary, as the example of the simple expression $\square \exists y(y=x)$ attests. Does it state that all possible objects are identical with something (a trivial logical tautology), or that actual objects exist in all possible scenarios (a far-from-trivial metaphysical claim)? It seems that we are free to choose which interpretation we want to follow, and one such choice is provided precisely by the modified translation scheme, as described earlier. The troublesome examples cited in the previous section may be put down to the mismatch between an assumed interpretation and an alternative one. For instance, the logical problem associated with the suggested interpretation of formula $\square \exists y[y=x \wedge \varphi(y)]$ may be explained away by pointing out that the meaning of the subformula $y=x$ within the range of the modal operator of necessity changes in comparison to the identity formulas applied outside such contexts. ${ }^{11}$ As a result, the formula in brackets is no longer logically equivalent to $\varphi(x)$, so there is no logical inconsistency in claiming that $\square \varphi(x)$ may have different truth conditions than the original formula.

Still, the situation is not entirely clear. As long as we use the same symbol $=$ for identity statements both inside and outside modal contexts, at least on the level of syntax the logical rule which allows us to exchange logically equivalent formulas salva veritate seems to be violated.

11 More precisely, the change of meaning is a result of both applying the modal operator and binding one variable while leaving the other free. In the case when both variables are free or both are bound, the meaning of the identity symbol may remain unaltered even within the range of a modal operator, as in the case of formula $\square \forall x \exists y(y=x)$. 
One possible solution could be to introduce a new symbol, for instance $\equiv$, to indicate that identity within modal contexts (and connecting a bound variable with a free one) is different from the normal one $=$. But then we are facing another thorny issue of how to interpret syntactically proper formulas in which the symbol $\equiv$ is used outside modal contexts (or in which it connects two bound variables). Either way, the necessary modifications of logic engendered by the adoption of the modified translation scheme may be seen as too high a price for the possibility of expressing various metaphysical conditions in the language of quantified modal logic.

\section{Cheap haecceitism}

One assumption of Lewis's [1968] counterpart theory is that objects possess no counterparts in the actual world other than themselves. This restriction has been removed in the 1986 variant of his theory, which bears the name of "cheap haecceitism" [Lewis 1986]. The reason for that modification of the original theory is that we should be able to account for the possibility of swapping actual objects without creating any qualitative difference. For instance, Lewis insists that it is possible for one of a pair of identical twins to be the other one. Given the standard analysis of possibilities in terms of possible worlds, accounting for such a possibility would require the existence of a world which would be qualitatively identical to the actual one, except that one twin would represent de re the other one, and vice versa. Since this would mean admitting haecceitistic differences not grounded in qualitative facts, Lewis argues that in this case alternative scenarios are represented not by possible worlds but by counterparts existing in the actual world. Thus one twin sibling is an actual counterpart of the other one, and represents a counterfactual situation in which both twins swap their identities.

It may be interesting to observe that adopting cheap haecceitism definitely precludes the possibility of using formula (2.4) as expressing the sufficiency condition, i.e., the condition that possessing the property denoted by $\varphi$ is a sufficient condition for being a counterpart of a given object. To see that, suppose that a particular actual object $a$ has some distinct counterparts in the actual world. In addition to that, let us suppose that all objects satisfying $\varphi$ across all possible worlds are counterparts of $a$ (thus the sufficiency condition (3.1) is fulfilled) Moreover, 
let us assume, as may very well be the case, that one actual counterpart of $a$ (call it $b$ ) such that $b \neq a$ satisfies $\varphi$. In that case, using simple rules of inference for modal logic, we can perform the following derivation:

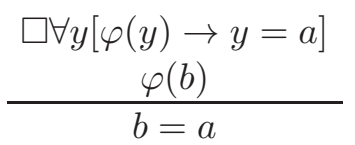

The conclusion of the above inference is false, and yet the premises are assumed to be true. This shows, quite independently of the specific translation rules for quantified modal logic proposed in Sec. 3, that Lewis's counterpart theory together with the assumption of cheap haecceitism is incapable of expressing the sufficiency condition in the form of the modal expression $\square \forall y[\varphi(y) \rightarrow y=x] .{ }^{12}$ The reason for this failure is rather simple: by introducing actual counterparts of objects we are 'mixing up' two types of identity: the numerical identity of objects within a given world and the 'counterfactual' identity expressed in the counterpart relation. And yet for a proper interpretation of the condition $\square \forall y[\varphi(y) \rightarrow y=x]$ we need to be able to read the identity formula within the range of the modal operator as expressing the counterfactual identity only.

\section{Conclusion}

Quantified modal logic provides a natural framework for expressing various statements discussed in the metaphysics of modality, including statements concerning essential properties of objects. In this brief commentary I have shown that we should exercise extreme caution when interpreting certain modal formulas involving quantifiers and the identity

12 In my [Bigaj 2016, p. 158] I used analogous reasoning which presupposes the modified translation scheme based on (3.2) as an argument against Lewis's cheap haecceitism. However, as we have observed in Sec. 3, even without cheap haecceitism the modified translation scheme is in trouble. On the other hand, the above result is not tied to any specific form of translation scheme, as its only crucial assumption is that the formula $\square \forall y[\varphi(y) \rightarrow y=x]$ expresses what we want it to express, i.e. the sufficiency condition (3.1). But the conclusion from the current argument is not so much the unconditional rejection of cheap haecceitism, as the impossibility of reconciling it with one intuitive interpretation of $\square \forall y[\varphi(y) \rightarrow y=x]$. Those who are happy with accepting the second horn of the dilemma (i.e., the rejection of the presupposition that this formula should express the sufficiency condition) may embrace Lewis's cheap haecceitism with no qualms. 
predicate. Within the broad framework of Lewisian counterpart theory it turns out that some intuitively appealing formulas, such as the 'sufficiency' formula $\square \forall y[\varphi(y) \rightarrow y=x]$, do not express what we want them to express. Some straightforward methods to alleviate this problem by modifying the rules of translation from quantified modal expressions into the language of counterpart theory create more problems than they solve. The problem with the intuitive interpretation of the above formula becomes even more egregious when we assume the variant of Lewis's counterpart theory known as cheap haecceitism.

One obvious lesson from this exercise is to avoid, if possible, using modal formulas as expressions of certain statements or assumptions in the metaphysics of modality. Instead, we can employ directly formulas of counterpart theory which are not in danger of misinterpretation, as suggested by Lewis himself in his [1986]. A downside of this strategy is that it commits us to a controversial metaphysical stance: the Lewisian theory of possible worlds and their inhabitants - possible objects. Some can consider this too high a price to pay. ${ }^{13}$ But an alternative is to rely on formulas whose intuitive, meaning diverges from their actual semantical interpretation and therefore give rise to conceptual confusion.

Acknowledgments. I would like to thank two anonymous referees for their deep and incisive comments on an earlier version of this article. The work on this paper was supported by grant No. 2017/25/B/HS1/00620 from the National Science Centre, Poland.

\section{References}

Bigaj, T., 2016, "Essentialism and modern physics", pages 145-178 in T. Bigaj and C. Wüthrich (eds.), Metaphysics in Contemporary Physics, Brill/Rodopi, Leiden-Boston. DOI: 10.1163/9789004310827_008

Fine, K., 1994, "Essence and modality", pages 1-16 in Philosophical Perspectives, vol. 8, Logic and Language.

13 See for instance [Skow 2008, 2011] for an expression of this position. This objection can be blunted though by noting that the transition to the framework of possible worlds does not have to commit us to full-blown Lewisian modal realism, but instead can be achieved within the more moderate position of so-called Ersatzism, as suggested by Stalnaker in [1976]. Regardless of which interpretation of possible worlds we prefer, one conclusion from the current paper is that the abandonment of the framework of possible worlds, counterparts etc. leaves us in a precarious position due to the notorious ambiguity of modal formulas involving identity. 
Garson, J. (2018), "Modal logic", in E. N. Zalta (ed.), The Stanford Encyclopedia of Philosophy, Fall 2018 Edition. https://plato.stanford.edu/ archives/fall2018/entries/logic-modal/.

Glick, D., 2016, "Minimal structural essentialism", pages 1-28 in A. Guay and T. Pradeau (eds.), Individuals Across the Sciences, Oxford University Press, Oxford. DOI: 10.1093/acprof : oso/9780199382514.003.0012

Lewis, D., 1968, "Counterpart theory and quantified modal logic", The Journal of Philosophy 65 (5): 113-126. Reprinted as [Lewis 1983]. DOI: 10.2307/ 2024555

Lewis, D., 1983, "Counterpart theory and quantified modal logic" with Postscript, pages 26-46 in Philosophical Papers, vol. I, Oxford University Press, Oxford. DOI: 10.1093/0195032047.003.0003

Lewis, D., 1986, On the Plurality of Worlds, Blackwell, Oxford.

Mackie, P., 2006, How Things Might Have Been. Individuals, Kinds, and Essential Properties, Clarendon Press, Oxford.

Skow, B., 2008, "Haecceitism, anti-haecceitism and possible worlds", Philosophical Quarterly 58: 98-107. DOI: 10.1111/j.1467-9213.2007.516.x

Skow, B., 2011, "More on haecceitism and possible worlds", Analytic Philosophy 52 (4): 267-269. DOI: $10.1111 / \mathrm{j} .2153-960 \mathrm{X} .2011 .00533 . \mathrm{x}$

Stalnaker, R., 1976, "Possible worlds", Nô̂s 10 (1): 65-75. DOI: 10.2307/ 2214477

Tomasz Bigaj

Faculty of Philosophy

University of Warsaw

Poland

t.f.bigaj@uw.edu.pl

https://orcid.org/0000-0002-8121-9789 